\title{
Reaction Times and Deception - the Lying Constant
}

\author{
Martin R. Sheridan \\ Department of Psychology, University of Hull \\ Hull HU6 7RX, UK \\ Tel: 44-1482-465-483Ｅ-mail: M.R.Sheridan@hull.ac.uk \\ Kenneth A. Flowers \\ Department of Psychology, University of Hull \\ Hull HU6 7RX, UK \\ Tel: 44-1482-465-388Ｅ-mail: K.A.Flowers@hull.ac.uk
}

\begin{abstract}
The cognitive theory of lie detection suggests that it takes longer on average to formulate a deliberately false response than a truthful one because it requires the truth to first be known and then altered, adding an extra component to the response process. This concept was upheld in a modified form in three experiments where subjects indicated as quickly as possible whether presented numbers were higher or lower than a given standard number, and to "lie" (give the wrong answer deliberately) on half the trials. Results suggested that lying adds a constant additional time to reaction times (RTs) independently of other factors such as the complexity of the cognitive task or method of response. Additionally, true Yes RTs were shorter than true No ones, producing an interaction with the lying constant such that RTs could reliably distinguish truth from lies for Yes responses but not so easily for No responses.
\end{abstract}

Keywords: Reaction time, Deception, Lying, Yes-no responses

\section{Introduction}

Attempts to distinguish between truthful and deliberately false (rather than just accidentally mistaken) responses to questions on the basis of their relative reaction times (RTs) go back to the beginning of the last century (Wertheimer, 1906) and were part of the first attempts to adapt experimental psychology techniques for a practical purpose, namely the detection of lying in criminal cases (Munsterberg, 1914.) The procedure used was an extension of the "association reaction" (that is, word association) method introduced by Galton (1879) and Cattell $(1887,1889)$ and standardised by Wundt (for review see Herbold-Wotten, 1982). The original method involved detecting "guilty knowledge" of a past event in suspects by comparing the words they produced as responses to critical probe stimulus words with similar responses produced by those without such knowledge, and with responses by the same guilty subjects to neutral words. Thus a neutral association to the stimulus "knife" might be "fork" but a guilty one might be "stab". This qualitative analysis was supplemented by measuring the RT to produce the response, where it was found that guilty responses to the critical probe words were often associated with hesitation, such that a relatively long RT could indicate guilt even when the association itself was innocuous. In fact it was thought that the extra time required to respond in these instances was due to the subjects' need to check their response before uttering it and to change it to an innocent one if necessary.

Thus developed the idea that increased reaction time could be used as an indication of lying or other guilt. Subsequent studies of the technique, however, weakened this idea, as, for example, when it was found that ordinary RT in word association is affected by a number of variables (such as concrete versus abstract nature of the words) and that subjects can be taught to lengthen or shorten their response times to critical words (Leach and Washburn, 1910). Also, it was difficult with individual responses to be sure that any delay was due to the lying factor and not to simple variation in RT. As other approaches to lie detection, such as physiological measures of arousal (Lykken, 1983; 1984; Gale, 1988), or the analysis of social cues (Ekman and Friesen, 1974; DePaulo, Stone, and Lassiter, 1985; Miller and Stiff, 1993) were developed this performance method of lie detection fell into disuse (although neither alternative method has proved very reliable either.)

More recently, however, the increased RT effect has been revived as a potential indicator of deceptive response where conditions are controlled appropriately (Dorward, 1999; Spence, Farrow, Herford, Wilkinson, Zheng \& 
Woodruff, 2001; Alban, 2003; Farrow, Reilly, Rahman, Herford, Woodruff \& Spence, 2003; Walczyk, Roper, Seeman, \& Humphrey, 2003; Vendemia, Buzan \& Simon-Dack, 2005). The critical feature of the method is that subjects should be uncertain of the exact nature of a truthful response to a question, so they cannot prepare the response in advance and have to process the information in the question in real time as quickly as possible, so that there is a cognitive processing load combined with a time stress in the performance of the task.

In these conditions the RT measure is a potentially reliable method of lie-detection because it exploits the natural characteristics of the information processing system and of immediate stimulus-response reaction behaviour which the voluntary behaviour system cannot accurately mimic deliberately and so are hard to fake. That is, it may be said that the "default mode" of the cognitive and perceptual-motor systems is to produce the correct or direct response to a given question or stimulus as quickly as possible, and any interference with that to produce a different incorrect or false response will always take slightly longer because it requires the natural response to be inhibited and another one to be deliberately constructed in its place. Either of these actions could be the source of increased RT, according to whether it reflects the increased cognitive load of the lying condition in constructing a false response, or that an extra decision operation has to be added on to the series of operations involved in the generation of the response. If it is the former, one might expect RT to vary according to the complexity of the task, whereas if it is the latter it would add a constant extra addition to RT. This investigation was intended to distinguish between these possibilities.

In the task presented here subjects were required to make either truthful or false (deliberately wrong) answers to standard questions, without knowing in advance either what the true answer to the question was or whether they would have to lie or not. Given that lying adds an extra load or component to whatever processes are involved in deciding the answer to a question, it was hypothesised that reaction times for false responses (RTf) would tend to be slightly longer than those for true ones (RTt). Because of natural variation in the time taken for the initial cognitive processes, this extra "lying time" may be difficult to discern in individual instances (unless it is very long) because it may be confounded with naturally long basic RTs, but it should show up reliably for average RT when samples of true and false responses are compared and the variability of these responses is allowed for. This prediction was tested in three experiments involving a simple numerical comparison task and requiring a simple Yes or No response, and deliberately wrong answers on half the trials. By regularising the cognitive task into a standard format which kept the form of the question the same for all trials across conditions, any variability of processing time related to question complexity would be controlled for, so that the comparison of true and false reactions was kept separate from variations in the cognitive task itself. In these conditions it was predicted specifically that on trials where subjects had to give a deliberately wrong answer to a standard question it would take them a constant added time to respond compared to trials where they gave correct answers to the question, and that this would be independent of other variables which affect reaction time.

\section{Experiment 1}

The reaction time method requires a task involving real-time cognitive calculations (choice reactions) which preclude anticipation of the exact question to be asked or the response to be made, otherwise subjects can prepare their responses in advance and thus reduce the task to one of simple reaction time. In this experiment the task on each trial was to compare a series of presented 2-digit test numbers with a prior given standard 2-digit comparison number (termed "your number" to the participant) and respond Yes or No in answer to one of two questions: "Is the next number greater than your number?" or "Is the next number less than your number?" Half the presented numbers were higher than the standard and half were lower, so the response Yes or No varied according to the question asked and the actual numbers involved. Each test number was presented with a letter T or L above it to indicate whether the response should be true or false, so again the response made had to be calculated in real time. It was predicted that RTs for false responses would on average be longer than those for true ones.

\subsection{Method}

\subsubsection{Participants}

13 undergraduate volunteers from the University of Hull were tested, 5 male and 8 female. All gave informed consent to take part after the nature of the task had been explained to them. Ages ranged between 18 and 30 years. All had normal or corrected-to-normal vision.

\subsubsection{Materials}

Stimuli were presented by means of an Electronics Developments Corporation 3-field tachistoscope and timer. The T-scope started the timer which was stopped by the participant pressing one of two keys, marked Yes and 
No. The number stimuli were presented on white cards in the centre of the visual field in Arial Black 36pt. (10 mm high) as were two question cards which read "Is the next number GREATER than your number?" or "Is the next number LESS than your number?"

\subsubsection{Procedure}

Participants were first given the target number 55 to remember as "their" number and told that they would be required to compare this number with other 2-digit numbers presented on the T-scope. They were told that on each trial they would be asked beforehand whether the presented number was either greater or less than their number, and that on half the trials they would be required to give the wrong (opposite) answer to the true one (that is, to lie), indicated by a letter L shown above the presented number, and on the other half of the trials they were to give the true answer, indicated by a letter T. To familiarise them with the task they were given initial practice trials, and then more if necessary until they were reliably producing responses appropriate to the $\mathrm{T}$ and $\mathrm{L}$ conditions. They were also assured that they could take a break at any time during the experiment.

Participants were then shown a series of 2-digit numbers from the ensemble 11-54 and 56-99 for 5 seconds each time (11 higher and 11 lower numbers in each of the 4 categories - Yes/True; Yes/Lie; No/True; No/Lie.) The numerical distance between the standard and test numbers was balanced equally across conditions. Prior to each presentation one of the two questions was shown for 4 seconds, followed by a fixation cross for 2 seconds. Participants pressed either a Yes key or a No key as quickly as possible, measured to the nearest ms. Above each stimulus number was a letter $\mathrm{T}$ (Truth) or $\mathrm{L}$ (Lie) to indicate whether the response should be true or false for that trial. The sequence of numbers, of Yes/No responses, and of $\mathrm{T}$ or $\mathrm{L}$ conditions were all balanced together in a pseudo-random order. Very few errors and responses longer than 5 seconds occurred, but where they did the stimulus was repeated at the end of the sequence so that a full set of correct responses was obtained for each condition.

\subsection{Results}

Reaction times for numbers above and below 55 were pooled for analysis, giving 22 responses per participant in each of the four categories (Yes true, Yes false, No true, No false.) These are shown in Fig.1A (where separate symbols are used for False Yes and False No responses to help clarify later

Discussion, see section 2.3.)

\section{Insert Figure 1 about here}

Analysis of variance showed a main effect between true and false responses $(F(1,11)=60.5, p<0.001)$ such that RTf (mean reaction time $1998 \mathrm{~ms}$ ) was overall significantly longer than RTt (mean reaction time $1570 \mathrm{~ms}$ ). There was also an interaction $(\mathrm{F}(1,11)=15.2, \mathrm{p}<0.01)$ between Yes and No and true and false conditions (shown in Fig.1). Subsequent Tukey HSD tests indicated that RTt Yes scores were faster than RTt No scores $(\mathrm{p}<0.05)$ and considerably faster than RTf Yes scores $(\mathrm{p}<0.001)$ but that RTt No scores were not reliably faster than RTf No scores $(\mathrm{p}>0.05)$. There was also no statistical difference between RTf reaction times for Yes and No responses $(\mathrm{F}(1,11)=0.34, \mathrm{~ns})$.

\subsection{Discussion}

The results of this experiment suggest two effects at work in the reaction times. First, participants are quicker when saying Yes truthfully than when saying No truthfully. Since Yes and No responses were equally distributed and thus equally probable across high and low numbers and across the "greater than" and "less than" questions asked, this effect is not likely to be an artefact of the procedure or of response expectation. On top of this, it takes longer to generate a false response than a true one whatever the calculation or response required, but the effect is apparently greater for Yes responses than for No ones, which means that the distinction between true and false Yes responses is marked, but that between true and false No responses is much smaller and not reliable.

This apparently paradoxical effect is explicable when one remembers that false responses are in fact the opposite true response with a lying factor added. Thus false No responses $(\mathrm{c} .1900 \mathrm{~ms})$ are really true Yes responses (c1400 ms) plus a delay of about $500 \mathrm{~ms}$, while false Yes responses $(\mathrm{c} .2100 \mathrm{~ms})$ are really true No responses (c.1700 ms) plus a delay of about $400 \mathrm{~ms}$ ). If the false response line on the graph is reversed to reflect this, it can be seen that there are two distinct effects in the data, a difference between true Yes and No responses, and a lying constant of somewhat over $400 \mathrm{~ms}$. added to it. This is shown in Fig. 1B, where the false Yes and No responses are reversed on the graph. It implies that, as predicted, the lying constant is independent of the cognitive processing involved in formulating answers to the questions put, although because of the reversal of actual response (Yes or No) in the task it appears that lying does interact with the generation of the underlying response to the question. 


\section{Experiment 2}

In experiment 1 participants responded by pressing keys with their hands. This raised the theoretical possibility that differences in RT for Yes and No responses were, at least in part, due to differences in response times between the hands (this variable was not recorded in experiment 1 as participants were allowed to press the Yes and No keys with whichever hand was convenient for them.) The possibility was unlikely, since the difference was some $400 \mathrm{~ms}$ and therefore several times larger than measured differences in response time between preferred and non-preferred hands, which are usually found to be less than $100 \mathrm{~ms}$ (Flowers, 1975). They are also larger than the movement initiation time of about $190-260 \mathrm{~ms}$ for movements in general (Carlton, 1992). Moreover, this could not invalidate the truth-lie difference as these responses were distributed equally between Yes and No responses. However, in order to check that the effects were independent of response mode, a second experiment was run replicating experiment 1 but with spoken responses and RTs measured with a voice key. In addition, response times for responses to the two questions (Is the next number GREATER than/LESS than your number?) were compared in the analysis, to see if the actual calculation affected or interacted with the Yes/No and truth/lie differences. Experiment 2 was otherwise the same as experiment 1 in design and procedure.

\subsection{Method}

\subsubsection{Participants}

12 undergraduate volunteers from the University of Hull were tested, 6 male and 6 female. Ages ranged between 19 and 30 years. All had normal or corrected-to-normal vision.

\subsubsection{Materials}

These were the same as in the previous experiment.

\subsubsection{Procedure}

This was the same as in experiment 1, except that participants spoke their answers into a microphone placed immediately below the viewing part of the T-scope, and reaction times were measured from the beginning of the exposure until the response was said.

\subsection{Results}

Reaction times for numbers above and below 55 were again pooled for analysis, giving 11 responses per participant for the two comparisons (greater than, less than) in each of the four categories (Yes true, Yes false, No true, No false.) Initial analysis of variance showed that the 3-way interaction between the greater-than and less-than questions factor and the truth/lie and yes/no ones was not significant $(\mathrm{F}(1,10)=5.54$, ns.) There was a non-significant difference in RT between the two types of question, with responses to greater-than questions (mean $=1464 \mathrm{~ms}$ ) taking slightly less time than those to less-than questions (mean $=1553 \mathrm{~ms}$.) The data for the two questions were therefore combined to give 22 responses in each of the four response categories. These are shown in Fig. 2.

\section{Insert Figure 2 about here}

Subsequent analysis of variance with combined responses showed a main effect between true and false responses $(\mathrm{F}(1,10)=37.99, \mathrm{p}<0.001)$ such that mean $\mathrm{RTf}(1695 \mathrm{~ms})$ was overall significantly longer than mean RTt (1322 ms.) There was also a significant interaction $(\mathrm{F}(1,11)=9.27, \mathrm{p}<0.05)$ between Yes and No and true and false conditions (shown in Fig.2.) The pattern of scores was similar to that of experiment 1. As before there was no overall main effect of Yes/No responses $(\mathrm{F}(1,10)=0.22$, ns) with mean RT for Yes reactions $(1498 \mathrm{~ms})$ being only slightly faster than that for No reactions $(1519 \mathrm{~ms}$.)

\subsection{Discussion}

Experiment 2 replicated the first experiment very closely, finding the same effects and interactions between true and false and Yes and No responses. Response times overall were faster than in the first experiment, probably due to the different mode of response employed, or possibly to the different participants taking part in the experiment. The pattern of reaction times in terms of the main truth/lie effect and the interaction of this with yes/no factor was the same as before. Thus the effect is independent of whether the response is manual or oral.

Previous studies have suggested that the increase in RT for lies varies according to the cognitive load of the task and/or of the response required (Cody, Marston \& Foster, 1984; Alban, 2003; Walczyk, et al., 2003.) In experiment 2, however, the nature of the question did not affect response times to any extent. Although there was a slight difference between reaction times for "greater than" and "less than" questions, it was not significant. This is not surprising, however, since the question format was standardised and there was no a priori reason to 
think that there would be a difference in cognitive load across conditions. To test for the effect of differing cognitive load in the underlying questions and whether this factor interfered with RTs for true and false Yes and No responses, a third experiment was performed involving two levels of complexity of the cognitive task.

\section{Experiment 3}

Experiment 3 was designed as a second replication of experiment 1but with two levels of complexity of the initial calculation built in to the task. This was done by posing the question to be answered in either a direct (positive) form or an indirect (negative) one. The latter, requiring more complex grammatical "decoding", was thought to require more processing in the calculation, and in a pilot study with true responses only, showed this to be true, as it was found to produce longer reaction times and to be subjectively more demanding. A sequence of 80 numbers was presented as before, with half the questions preceding each number in the simple format and half in the more complex one.

\subsection{Method}

\subsubsection{Participants}

12 undergraduate volunteers from the University of Hull were tested, 6 male and 6 female. Ages ranged between 19 and 30 years. All had normal or corrected-to-normal vision.

\subsubsection{Materials}

These were the same as in the previous experiment, except that four question cards were used:

Is it TRUE that the next number is GREATER than your number?

Is it NOT TRUE that the next number is GREATER than your number?

Is it TRUE that the next number is LESS than your number?

Is it NOT TRUE that the next number is LESS than your number?

The stimuli comprised the 80 two-digit numbers from 10 - 49 and 60 - 99, half of which were presented with a T above them and half with an $\mathrm{L}$. The four questions and two truth/lie conditions were pseudo-randomly distributed in a counterbalanced order across the high and low numbers, giving 10 responses in each of the 8 conditions for each participant. Not more than four consecutive correct Yes or No responses occurred in sequence.

\subsubsection{Procedure}

This was the same as in experiment 2, with participants speaking their answers to a microphone immediately under the T-scope. Reaction times were measured from the beginning of the exposure until the response was said. Again there were only a few trials with errors or response times greater than 10 seconds, and these were repeated at the end of the sequence so as to obtain a full set of responses in each condition.

\subsection{Results}

Reaction times for numbers above and below 55 were again pooled for analysis, giving 10 responses per participant in each of the four categories (Yes true, Yes false, No true, No false) for the two conditions (simple and complex.)

Analysis of variance showed that there was a main effect of complexity of the question $(F(1,10)=22.80$, $\mathrm{p}<0.001)$ but that there was no interaction between this factor and either truth/lie responses $(\mathrm{F}(1,10)=0.94, \mathrm{~ns})$ or Yes/No responses $(\mathrm{F}(1,10)=1.10, \mathrm{~ns})$ nor was there any 3 -way interaction between these three factors $(\mathrm{F}(1,10)$ $=1.25$, ns.) Thus responses to the more complex question took longer (mean $3622 \mathrm{~ms}$ overall) than those to the simpler one (mean $2481 \mathrm{~ms}$ ) but this was independent of the other influences on reaction time. Thus, importantly, the nature of the interaction between

Yes/No and true/false was not altered by the complexity of the question, even while the increased complexity of the basic task resulted in an increase in RTs overall compared to the first two experiments.

There was a main effect of truth/lie conditions $(\mathrm{F}(1,10)=140.30, \mathrm{p}<0.001)$ but not between Yes and No responses $(\mathrm{F}(1,10)=1.66$, ns.) The interaction between these did not quite reach conventional significance $(\mathrm{F}(1,10)=3.81, \mathrm{p}<0.08)$, probably because with the extra factor of complexity there are only 10 responses in each of the 8 categories, but the pattern of responses was the same as in the previous experiments. These are shown in Fig. 3. 
Another main effect found post-hoc in the data was a general reduction in RT across trials, that is, responses became faster with practice through the task. This effect was significant $(F(9,90)=6.50, p<0.001)$ but again it did not interact with complexity $(\mathrm{F}(9,90)=0.56$, ns), nor with truth/lie $(\mathrm{F}(9,90)=1.11$, ns), nor with Yes/No responses $(F(9,90)=2.01$, ns.) The relative RTs for Yes and No true and false responses, and for cognitive processing of differing levels of difficulty, therefore, are preserved even as practice improves response rate.

\subsection{Discussion}

The pattern of response times for true and false and for Yes and No responses in this experiment replicated that found in experiments 1 and 2, and was independent of the complexity of the underlying cognitive task, and of any practice effect. Reaction times were higher overall in this experiment, probably reflecting the increased cognitive load of the number calculation even for the simpler question, and there was a main effect of question complexity, but these did not interact with the lying constant, nor with difference between Yes and No response, both of which are separate characteristics of the comparison process.

\section{General Discussion}

The conclusion from Experiment 1 was that all deliberately false responses take significantly longer to generate on average than true ones, but that this lengthening is different for false Yes and false No RTs, with the latter only slightly longer than their true equivalents while the former are more obviously longer. This apparent discrepancy is resolved when it is realised that false responses in fact comprise the opposite true response plus an extra delay for the reversing of the response, and that there is a significant difference (on this task at least) between true Yes and true No responses in the first place. When this is allowed for two separate RT effects appear, firstly, a difference between responding Yes and No, and secondly, a longer response time for false responses compared to true ones. This latter effect is a constant.

Experiment 2 replicated this pattern of results in a similar task where responses were spoken rather than manual, showing that the effect is in the cognitive (decision-making) aspect of the task independently of the mode of generation of the response.

Experiment 3 also replicated the pattern of results in a task where the complexity of the original decision-making task was increased, so that underlying response time was longer. The importance of these results was to demonstrate that the extra time involved in generating true responses adds to the overall RT but that this is independent of the extra time required to reverse a true response deliberately, which is added on to whatever calculation time is required by the question, and is a constant.

Thus, independently of whatever cognitive processes are involved in generating responses, and of the mode of generating the response itself, lying adds a constant delay to true responses, and can be reliably distinguished from true responses in terms of average RT. This is the "lying constant."

That saying Yes is faster than saying No in genuine (truthful) responses is a curious effect as yet unexplained, but, from the evidence here, robust. Assuming that it is based on a difference in calculation time for the two responses rather than any "conforming" tendency in the decision process, it suggests that mentally comparing the two numbers must allow a Yes response to be arrived at with less cognitive processing than a No response. A possible explanation would be that when presented with the stimulus number subjects begin some internal scanning process to compare their number with the target, and if a match is found they can say Yes, whereas only when no match is found can they say No. Alternatively, the priming question may direct internal attention in some way to the ensemble of numbers either above or below the target number (or to the "left" or "right" in a spatial number set), allowing a quicker match if there is one with the relevant number than if the number does not fall within that ensemble. It does seem clear, however, that the comparison process is not a matter of simple subtraction or numerical comparison, since if it was, the answer Yes or No should be on average equally fast. The reason for the difference merits further investigation.

Whatever the basis of the Yes-No effect, it produces a false apparent interaction with the True-Lie effect which obscures the second effect found here, the constant increase in reaction time associated with lying. That is, the extra time taken to generate a false response is the same for both responses of the Yes/No type and is added to whatever time is needed to process the underlying question and prepare a correct response. When the genuine Yes-No difference is separated out, the lying constant appears reliably. The confusion may not have been realised before because tasks that require more than a simple Yes or No response introduce another source of variation in response time which confounds these two effects further. Restricting the questions to the formula adopted here may allow the effects to show up and answer one of the criticisms of this approach which led to the 
method being dropped in the past, namely that variation in underlying response time obscures the difference in time between true and false responses.

That lying involves an increase in response time has, as noted in the introduction, been known for well over a century, and has been regularly confirmed in more recent studies (Dorward, 1999; Spence et al, 2001; Alban, 2003; Farrow et al., 2003; Vendemia et al., 2005). The reason for this is, however, in dispute. The most frequently advanced concept is that lying increases the cognitive load of respondents (Cody et al., 1984; Alban, 2003; Walczyk et al., 2003) so that effective deceptive messages require more cognitive effort to produce than truthful ones. Deceivers must first construct messages that are consistent with existing facts and therefore a plausible substitute for the truth. All subsequent messages must also be monitored to make sure that they are consistent. Cody et al. (1984) identified a number of vocal and verbal cues, of which response time was one, that could be used to tell liars from truth-tellers. Alternatively, Farrow et al. (2003) suggest that the delay is due to the need to inhibit the natural response before producing the false one, that is, it requires a separate extra stage in the sequence of operations involved in generating the response. Our results are more in line with this suggestion, in that the extra time for false responses was found to be independent of the cognitive load for the basic calculation. Thus lying appears to add a specific extra cognitive operation to the generation of the response, rather than increasing the overall cognitive load of the calculation itself. Farrow et al. (2003) also claimed that the effect is independent of the mode of presentation (auditory or visual).

Each response in the present task, however, was a separate "answer", with no connections in meaning between successive questions, so the participants did not have to keep track of their previous responses when deciding how to formulate the current one. Also, the decision whether subjects should tell the truth or lie each time in this study was made by the experimenter when presenting the stimulus number, so they were relieved of the need to decide whether to lie, having only to decide how to do so. The cognitive load in this task, therefore, may have been less than in that of Cody et al. (1984), Walczyk et al. (2003) and other such studies, particularly as the response required is fixed and simple so that subjects have only to choose one of two alternatives. The lying constant may thus be a feature of "closed" tasks, and represents the minimum time required to reverse a response. Where more creative or complicated responses are required, the time taken is likely to be progressively longer and to reflect the processing load involved in elaborating them. In experiments where the task resembles ours here (that is, constant unelaborated Yes-No responses) the increase in RT for false responses is uniformly reported to be around 200-230 ms (Farrow et al., 2003; Walczyk. 2003, in Yes/No condition; Vendemia et al., 2005)

Previous studies have not distinguished between Yes and No responses, and so have not found the interaction that is the most striking aspect of the results here. Vendemia et al. (2005) found a similar effect in experiments where subjects responded "true" or "false" to statements, which is an equivalent condition, but only where both the statement to be evaluated and the instruction to tell the truth or lie were given simultaneously, as in this study. They comment only that this condition must affect attention in some way. We suggest a more specific explanation for the interaction, which shows up only when subjects cannot anticipate the nature of their response, that is, they do not know which response, Yes or No, will be the truthful one and which the lie. Here, if subjects know in advance they will be lying, they can simply reverse the question asked and then reply to the number truthfully, in which case they can "lie" as quickly as they can respond truthfully. Thus, if you are going to lie to the question "Is the next number GREATER than your number?" and the number is 20 (true answer therefore No) one need only reformulate the question as "Is the next number LESS than your number?" and reply Yes. Similarly, if the number is known in advance, subjects can formulate the two possible answers (True - say Yes; Lie - say No) and simply produce the one appropriate to the instruction when it appears. Only when the underlying calculation and the strategy have to be determined together does the interaction (and the reliable lying constant) appear consistently.

The evidence here from all three experiments suggests that subjects are quicker when saying Yes truthfully than No truthfully, but are slower to lie saying Yes than No. Thus, while false responses generally are slower than truthful ones the effect is much greater when subjects are saying Yes. Efficient lie detection, therefore, may depend on structuring the response situation in such a way that the subject is required to produce Yes answers (thus, "Are you innocent?" rather than "Are you guilty?") Subjects should have to respond Yes to a critical question a number of times and their RTs compared to those for questions where the known true answer is Yes. Interestingly, this technique may constitute more of a "truth-detection" rather than a lie-detection test, in that, if subjects can produce Yes responses fast enough where Yes is the "innocent" answer, it is highly likely that they are telling the truth, whereas if they do not it may be equivocal whether they are lying. Also, if hesitancy is used 
as a major cue to distinguish true from false responses in social situations, it may be difficult for observers to tell whether No responses are genuine or false, which could be a factor in social misunderstandings.

Another feature of the lying constant is that it shows up as an average increase in RT between two samples of responses. Individual response times cannot reliably distinguish between them since other sources of variation in RT may well obscure the relatively modest $200-400 \mathrm{~ms}$ difference. The effect found here, therefore, requires repeated answers to questions formulated in such a way that the intended answer involves some uncertainty and therefore some on-line cognitive processing repeatedly in a random sequence so that prepared responses cannot be easily used. Any test based on response times, therefore, would have to include such constraints for it to produce evidence of whether someone is telling the truth or not based on the lying constant. If such a test could be formulated it might answer another of the criticisms which led to the abandonment of the approach as a practical method of investigating the truthfulness of accused or witnesses a century ago, that is, that it is possible to sabotage the test by preparing false responses in advance and so bypass the increased reaction time normally associated with lying. Whether this is practical remains to be determined.

Participants in this investigation were not given any incentive to beat the test by trying to disguise their false answers so as to make them indistinguishable from true ones. If the default mode of the nervous system is to produce correct (true) responses as quickly as possible (since in most normal situations this would be desirable for effective action) then all false responses which require some change to these processes will involve an added delay and hence longer reaction time. The only way such false responses could be hidden, therefore, would be by adding a delay to true answers so as to make them equally slow. However, although this strategy may hide the hesitation in false reactions in terms of overall mean, we have found in some preliminary investigation (not yet reported) that it alters the distribution of true reaction times in a number of other ways too, which may indicate equally clearly that performance is abnormal. Thus not only is there an increase in the mean of RTs but also an increase in the variance, and the normal shape of the distribution is destroyed, so that it is clear from the pattern of true response times alone that the participant is not responding naturally. This is then as good an indication of suspicious behaviour as the lying constant in this experiment.

While such analysis can never in itself directly determine that a response is false, it does suggest that it is suspicious. Interestingly, however, it may be that reaction time tests of this kind could have an opposite role, that of establishing innocence rather than guilt. If a suspect can produce a set of responses to crucial probe questions with a genuine range of reaction times within a normal-shaped distribution with a mean equal to that of other neutral questions, then they must be "innocent", since on our evidence this should not be possible if they are lying. (If they cannot do this, or reaction times are all over the place, then they may be guilty but may also merely be being abnormally careful or just disturbed.) The task may, therefore, work better as a truth-detection test rather than a lie-detector, and it may be more fruitful to determine the characteristics of truthful responses than false ones as a basis for distinguishing between them. To do this, one may speculatively suggest that a test should require a series of questions for subjects to respond to with the characteristics:

1. questions to be structured in such a way as to meaningfully demand either a Yes or No answer; but

2. whether the answer to each question is Yes or No be not known in advance, so some cognitive processing of the question on-line is necessary;

3. crucial questions have to be mixed in with neutral or irrelevant questions also requiring responses as fast as possible;

4. at least some of the innocent answers to crucial questions should require a Yes response so that the maximum difference between genuine and false responses will show up;

5. crucial questions must be repeated so as to produce a sample of responses so that an average RT can be calculated and the probability of it being longer than genuine ones determined.

Such a battery questions may appear to be bizarre, but is not that different from repeated questioning techniques reportedly used in interrogations, and can be easily automated so as to reduce the emotional atmosphere of questioning, and the test could even be self-paced to reduce the external distraction involved in face-to face questioning.

\section{References}

Alban, A.D. (2003). Neuropsychological malingering and cognitive load: disrupting deception of neuropsychological measures through cognitive overload. Dissertation Abstracts International, Section B, 63 (9-B), 4358. 
Carlton, L.G. (1992). Visual processing time and the control of movement. In L.Proteau \& D.Elliott (Eds.), Vision and Motor Control (pp.3-31). Amsterdam: Elsevier.

Cattell, J.McK. (1887). Experiments on the association of ideas. Mind, 12, 68-74.

Cattell, J.McK. (1889). Mental association investigated by experiment. Mind, 14, 230-250.

Cody, M.J., Marston, P.J., \& Foster, M. (1984). Deception: Paralinguistic and Verbal Leakage. In R.N.Bostrom (Ed.), Communication Yearbook 8. (pp. 464-490). Beverly Hills, CA: Sage.

De Paulo, B.M., Stone, J.I., \& Lassiter, G.D. (1985). Deceiving and Detecting Deceit. In B.R.Schlenker (Ed.), The Self and Social Life (pp.333-370). New York: McGraw-Hill.

Dorward, J. (1999). Feigned recognition deficits and response latency as measures of defensiveness.Dissertation Abstracts International, Section B, 60 (6-B) 2971.

Ekman, P., and Friesen, W.V. (1974). Detecting deception from the body or face. Journal of Personality and Social Psychology, 29, 288-298.

Farrow, T.F.D., Reilly, R., Rahman, T.A., Herford, A.E., Woodruff, P.W.R., \& Spence, S.A. (2003). Sex and personality traits influence the difference between time taken to tell the truth or lie. Perceptual and Motor Skills, 97, 451-460.

Flowers, K.A. (1975). Handedness and motor control. British Journal of Psychology, 66, 39 -52.

Gale, A. (Ed.) (1988). The Polygraph Test: Lies, Truth and Science. London: Sage.

Galton, F. (1879). Psychometric experiments. Brain, 2, 149-162.

Herbold-Wotten, H. (1982). The German Tatbestandsdiagnostik. A Historical Review of the Beginnings of Scientific Lie Detection in Germany. Polygraph, 11, 246-257.

Leach, H.M., and Washburn, M.F. (1910). Some tests by the association reaction method of mental diagnosis. American Journal of Psychology, 21, 162-167.

Lykken, D.T. (1983). In A.Gale \& J.A.Edwards (Eds.), Physiological Correlates of Human Behavior. Vol.1: Basic Issues (pp.243-256). London: Academic Press.

Lykken, D.T. (1984). Polygraphic interrogation. Nature, 307, 681-684.

Miller, G.R., \& Stiff, J.B. (1993) Deceptive Communication. Newbury Park, CA: Sage.

Munsterberg, H. (1914). Grundzuge der Psychotechnik. Leipzig: J.A.Barth.

Spence, S.A., Farrow, T.F.D., Herford, A.E., Wilkinson, I.D., Zheng, Y., \& Woodruff, P.W.R. (2001). Behavioural and functional anatomical correlates of deception in humans. Neuroreport: for Rapid Communication of Neuroscience Research, 12, 2849-2853.

Vendemia, J.M.C., Buzan, R.F., \& Simon-Dack, S.L. (2005). Reaction time of motor responses in two-stimulus paradigms involving deception and congruity with varying levels of difficulty. Behavioural Neurology, 16, 25-36.

Walczyk, J.J., Roper, K.S., Seemann, E., \& Humphrey, A.M. (2003). Cognitive mechanisms underlying lying to questions: response time as a cue to deception. Applied Cognitive Psychology, 17, 755-774.

Wertheimer, M. (1906). Experimentelle Untersuchungen zur Tatbestandsdiagnostik. Inaugural Dissertation, Wurzburg. Archiv fur die gesamte Psychologie, 6, 59-131. 


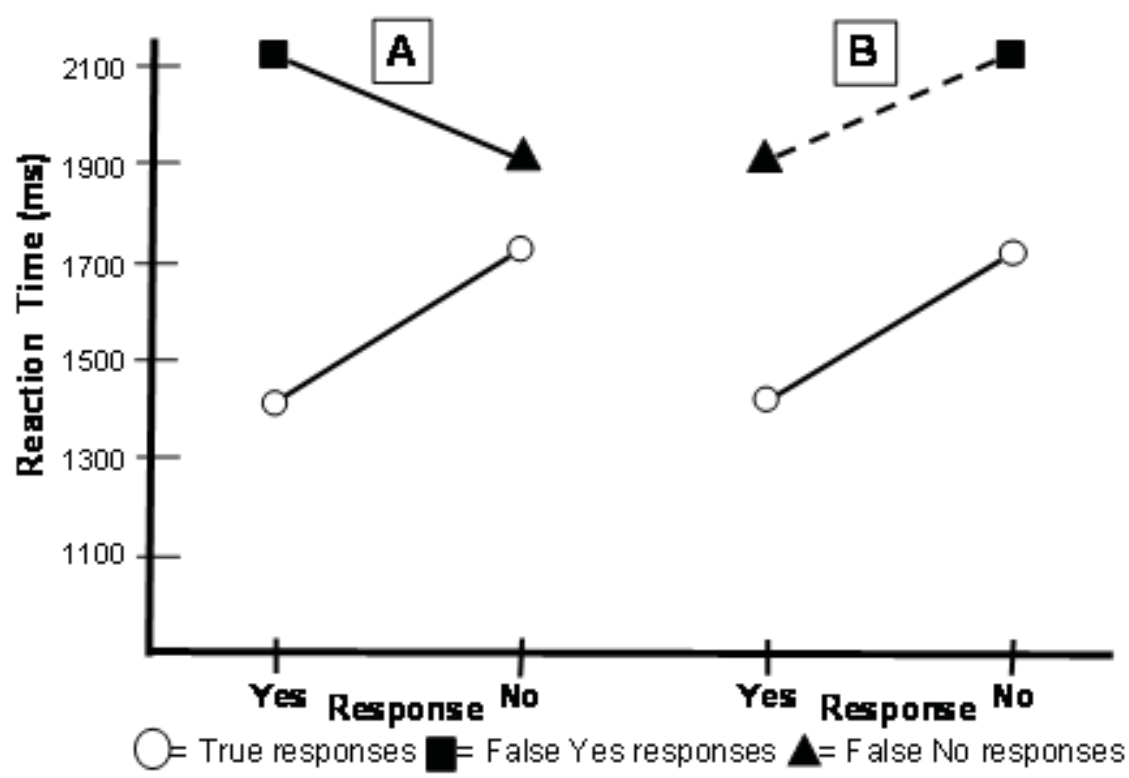

Figure 1. Experiment 1. Mean reaction time as a function of True/False conditions by Yes/No responses. 1A: original data. 1B: with False reaction times reversed (for explanation see text.)

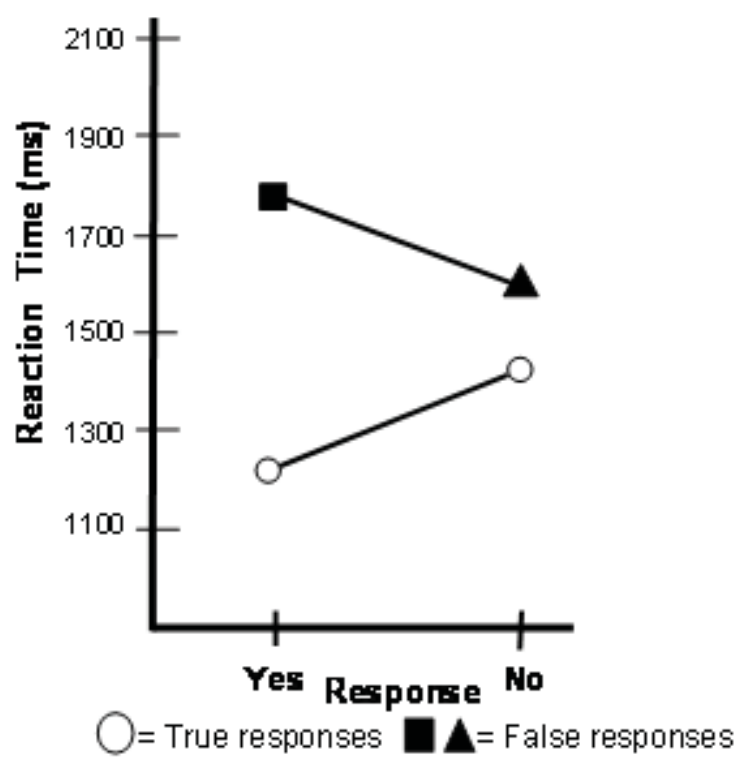

Figure 2. Experiment 2. Mean reaction time as a function of True/False by Yes/No responses 


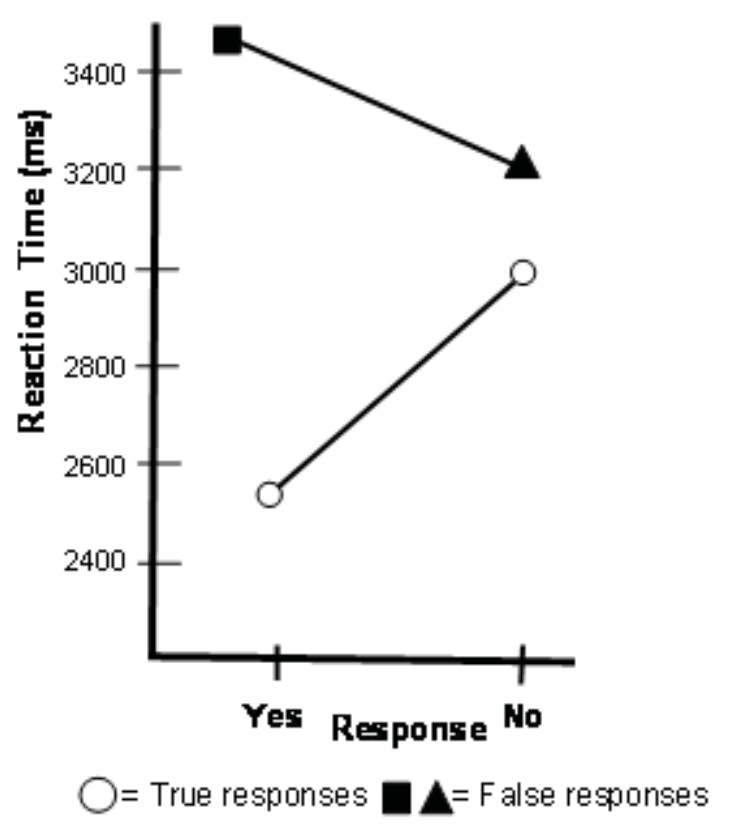

Figure 3. Experiment 3. Mean reaction time as a function of True/False by Yes/No responses 